\title{
IMPACTOS DOS BTEX EM ÁREAS URBANAS DA CIDADE DO RIO DE JANEIRO
}

\author{
Josie Batista Bastos Carvalhoa,*,®, Júlio Domingos Nunes Fortes ${ }^{\mathrm{a}}$, Sergio Machado Corrêa ${ }^{\mathrm{b}}$ e Eduardo Monteiro Martins ${ }^{\mathrm{a}}$ \\ ${ }^{a}$ Faculdade de Engenharia, Universidade do Estado do Rio de Janeiro, 20550-900 Rio de Janeiro - RJ, Brasil

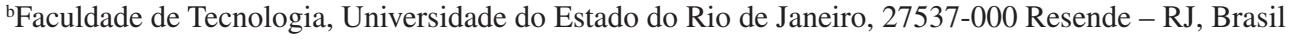

Recebido em 27/01/2020; aceito em 20/04/2020; publicado na web em 10/06/2020

\begin{abstract}
BTEX IMPACTS IN URBAN AREAS OF THE CITY OF RIO DE JANEIRO. Most volatile organic compounds (VOC) have adverse effects on human health and the environment and react individually with different velocities and mechanisms to form ozone. The present work determined the concentration of 55 samples of benzene, toluene, ethylbenzene and xylenes (BTEX), an important subgroup of VOC, by active sampling with active carbon cartridge in the areas of Barra da Tijuca, Deodoro and Maracana between the months of December 2014 to September 2015. The samples were extracted with dichloromethane and analyzed by gas chromatography coupled to mass spectrometry (GC-MS). The percentage distribution of BTEX showed toluene as the most abundant compound in the three sites, with 54,3\% in Barra da Tijuca, 57,4\% in Deodoro and 38,1\% in the Maracana area of the total BTEX. The mean benzene/toluene (B/T) ratios were 0.12 (Maracana), 0.37 (Barra da Tijuca) and 0.23 (Deodoro), confirming the emission source as predominantly vehicular. Using reactivity scales, toluene was the compound that most contributed to the formation of ozone and benzene was less reactive than the others. The carcinogenic risk was considered indifferent in all sites.
\end{abstract}

Keywords: atmospheric pollutants; volatile organic compounds; ozone; BTEX.

\section{INTRODUÇÃO}

Os compostos orgânicos voláteis (COV), em sua maioria, apresentam diversos efeitos adversos à saúde humana, são altamente reativos, possuem caráter tóxico, além de serem precursores do ozônio. ${ }^{1,2}$ Dentre os diversos COV existentes na atmosfera, destaca-se o subgrupo dos compostos monoaromáticos formados pelo benzeno, tolueno, etilbenzeno e xilenos (BTEX), que são predominantemente emitidos para a atmosfera pelo processo de combustão e evaporação dos combustíveis e processos industriais. ${ }^{3-6}$

$\mathrm{O}$ efeito da reatividade dos COV passou a ser reconhecido a partir da década de 90, em que foi possível perceber que o uso de COV menos reativos poderia levar a uma redução na concentração do ozônio. ${ }^{7}$ No cálculo da escala de reatividade, que é baseada na classificação dos COV em função do seu potencial de contribuição para a formação de ozônio, diversos parâmetros têm sido usados, como taxas de reação, rendimento do produto e os efeitos observados na razão COV/NOx em presença de radiação solar. ${ }^{8}$

A reatividade de um COV pode ser analisada através de suas reatividades cinética e mecanística. A primeira corresponde à velocidade de reação de um COV na atmosfera, relacionando o tempo de vida e a sua capacidade de oxidação. É geralmente obtida através do coeficiente de velocidade para a reação com radical hidroxila $\left(\mathrm{k}_{\mathrm{OH}}\right)$ e a concentração média de cada COV. Essa escala indica o impacto inicial de cada composto, mas desconsidera alguns fatores importantes, como os mecanismos de reações fotoquímicas. Já a reatividade mecanística utiliza o mecanismo completo, relacionando o potencial de formação de ozônio de cada COV com o coeficiente de velocidade $\left(\mathrm{k}_{\mathrm{OH}}\right)$ e o mecanismo de reação., ${ }^{7,-11}$

Carter $^{7}$ estabeleceu várias escalas para quantificar o impacto de um COV sobre a formação de ozônio $\left(\mathrm{O}_{3}\right)$. A escala MIR (Maximum Incremental Reactivity) é uma das mais conhecidas, onde cada COV possui um potencial MIR. Essa escala considera que um composto possui a mesma reatividade sob diferentes condições meteorológicas. Ao multiplicar a concentração de um determinado COV pelo

*e-mail: josiebsilva@gmail.com seu potencial MIR, o resultado obtido são as gramas de $\mathrm{O}_{3}$ que será formada por cada 1 grama de $\operatorname{COV}\left(\mathrm{O}_{3(\mathrm{~g})} / \mathrm{COV}_{(\mathrm{g})}\right)$.

Outras duas escalas de reatividade mecanística foram desenvolvidas por Carter: ${ }^{11}$ MOIR (Maximum Ozone Incremental Reactivity), que relaciona a concentração de NOx capaz de gerar a concentração máxima de $\mathrm{O}_{3}$ num determinado cenário e a escala EBIR (Equal Benefit Incremental Reactivity), na qual as concentrações de COV e $\mathrm{NO}_{\mathrm{x}}$ possuem a mesma efetividade na geração de ozônio.

Os BTEX possuem alta pressão de vapor, logo a via pulmonar é o meio preferencial de absorção destes compostos. O benzeno é classificado como carcinogênico e causador de leucopenia, enquanto que os demais compostos (tolueno, etilbenzeno e xilenos) são compostos neurotóxicos. ${ }^{4,12}$ Estudos mostram que a exposição ao ar interior e exterior pode ocorrer por inalação, ingestão ou absorção dérmica, porém a via predominante é a inalação. ${ }^{13-15} \mathrm{~A}$ exposição humana ao benzeno ocorre principalmente por inalação no local de trabalho, por emissões automotivas e fumaça de tabaco. ${ }^{12}$

O risco de câncer ocorre após exposição prolongada a um potencial carcinógeno dentro de um cenário, de acordo com os diversos fatores de exposição. ${ }^{16}$ Segundo a Agência Internacional de Pesquisa sobre o Câncer (IARC), ${ }^{17}$ os BTEX possuem uma classificação quanto ao risco para a saúde humana. Devido ao seu potencial em aumentar o risco de câncer, como por exemplo a leucemia, o benzeno é classificado como Grupo 1 (carcinogênico para humanos). Os demais compostos não são classificados quanto à sua carcinogenicidade para humanos, embora possuam potencial tóxico não carcinogênico.

A cidade do Rio de Janeiro, assim como qualquer outro grande centro urbano, sofre com as altas concentrações de ozônio, sendo refletido com mais intensidade em áreas próximas a grandes vias de tráfego veicular, em bairros muito urbanizados e distantes do mar. Dessa forma, é de fundamental importância conhecer a concentração dos BTEX, assim como de outros COV nas diferentes áreas da cidade, avaliar os potenciais de formação de ozônio, assim como os impactos na saúde. As características geográficas do Rio de Janeiro potencializam os efeitos relacionados à degradação da qualidade do ar, uma vez que a distribuição e dispersão dos poluentes podem ser prejudicadas por algumas barreiras físicas naturais como o maciço 
da Pedra Branca e da Tijuca, que podem influenciar diretamente na circulação dos ventos predominantes vindos do mar. ${ }^{18}$

Este trabalho tem como objetivo analisar a concentração de BTEX em três diferentes pontos da cidade do Rio de Janeiro, investigando o potencial de formação de ozônio a partir de uma classe de COV e avaliar o risco da exposição à saúde humana.

\section{PARTE EXPERIMENTAL}

\section{Área de estudo}

A cidade do Rio de Janeiro possui uma área de 1.197,463 km² de extensão e é banhada ao sul pelo Oceano Atlântico, ao leste pela Baía de Guanabara e a oeste pela Baía de Sepetiba. Sua topografia é caracterizada por planícies litorâneas cercadas de morros, baías, restingas, serras, lagoas e ilhas. Possui clima tropical, variando entre quente e úmido no verão, enquanto no inverno apresenta temperaturas amenas com períodos restritos de chuva. Sua temperatura média anual é de $23^{\circ} \mathrm{C}$ e o índice de precipitação varia em torno de 1200 a $1800 \mathrm{~mm} \cdot{ }^{19,20} \mathrm{~A}$ cidade possui uma população de aproximandamente 6,7 milhões de habitantes, com densidade demográfica de 5.265 habitantes por $\mathrm{km}^{2} \mathrm{e}$ frota veicular estimada em 2,9 milhões. ${ }^{19,21}$ A Figura 1 apresenta os três locais onde foram realizadas as coletas das amostras.

$\mathrm{Na}$ área da Barra da Tijuca (ou Barra), as coletas dos BTEX foram realizadas a uma distância de $1,22 \mathrm{~km}$ do Riocentro, $2,58 \mathrm{~km}$ do Parque Olímpico e em frente à Vila Olímpica. Na área Maracanã foram coletadas amostras na Universidade do Estado do Rio de Janeiro (UERJ), próximo ao Estádio do Maracanã e, em Deodoro, a amostragem foi realizada na Escola de Artilharia de Costa e Antiaérea (EsACosAAe), na região da Vila Militar, que fica localizada a $1,78 \mathrm{~km}$ de distância do Complexo Esportivo de Deodoro,

\section{Metodologia}

O período de amostragem ocorreu entre 8 e 17 horas, a fim de abranger os horários de maior tráfego veicular. As coletas foram realizadas nas áreas da Barra da Tijuca e do Maracanã entre dezembro de 2014 a agosto de 2015, em diferentes dias, abrangendo as estações verão, outono e inverno. Na área de Deodoro, o período de amostragem ocorreu entre abril e agosto de 2015, compreendendo as estações outono e inverno. A maior parte das coletas ocorreram em dias de sol intenso, com temperaturas variando entre 25 e $38^{\circ} \mathrm{C}$. Em média, foram coletadas duas amostras por mês nas áreas da Barra da Tijuca e de Deodoro. Na área do Maracanã, devido à logística favorável, foi obtido o dobro de amostras por mês. No total foram coletadas 28 amostras no Maracanã, 14 amostras na Barra da Tijuca e 13 amostras em Deodoro.

O método de referência utilizado para a amostragem de BTEX foi o NIOSH 1501 (National Institute of Occupational and Safety Health), ${ }^{22}$ com adaptações, em que foram utilizados cartuchos de carvão ativado com leito duplo (SKC 226-01), que eram acoplados a um tubo flexível conectado à bomba amostradora (Gillan, modelo BDXII - Sensidyne), com rotâmetro acoplado e faixa de vazão de trabalho de $0,8 \mathrm{~L} \mathrm{~min}^{-1}$. O tempo de amostragem foi de 6 horas por dia e a bomba era fixada em um tripé a 1,5 m da altura do solo para simular a respiração humana. ${ }^{3}$ A definição do tempo de amostragem torna-se importante a fim de obter um limite de detecção adequado para o método analítico sem que haja saturação do leito principal do cartucho. ${ }^{23,24}$ Após a amostragem, os cartuchos foram lacrados com as tampas apropriadas, envoltos em papel alumínio e transportados sob refrigeração até o laboratório para posterior análise. A estabilidade da amostra é mantida por até 30 dias após a coleta sob temperatura de $5{ }^{\circ} \mathrm{C} .{ }^{22}$ Os ensaios em branco eram realizados a cada coleta e os cartuchos foram tratados da mesma forma que a amostra, porém sem a passagem de ar através da bomba.
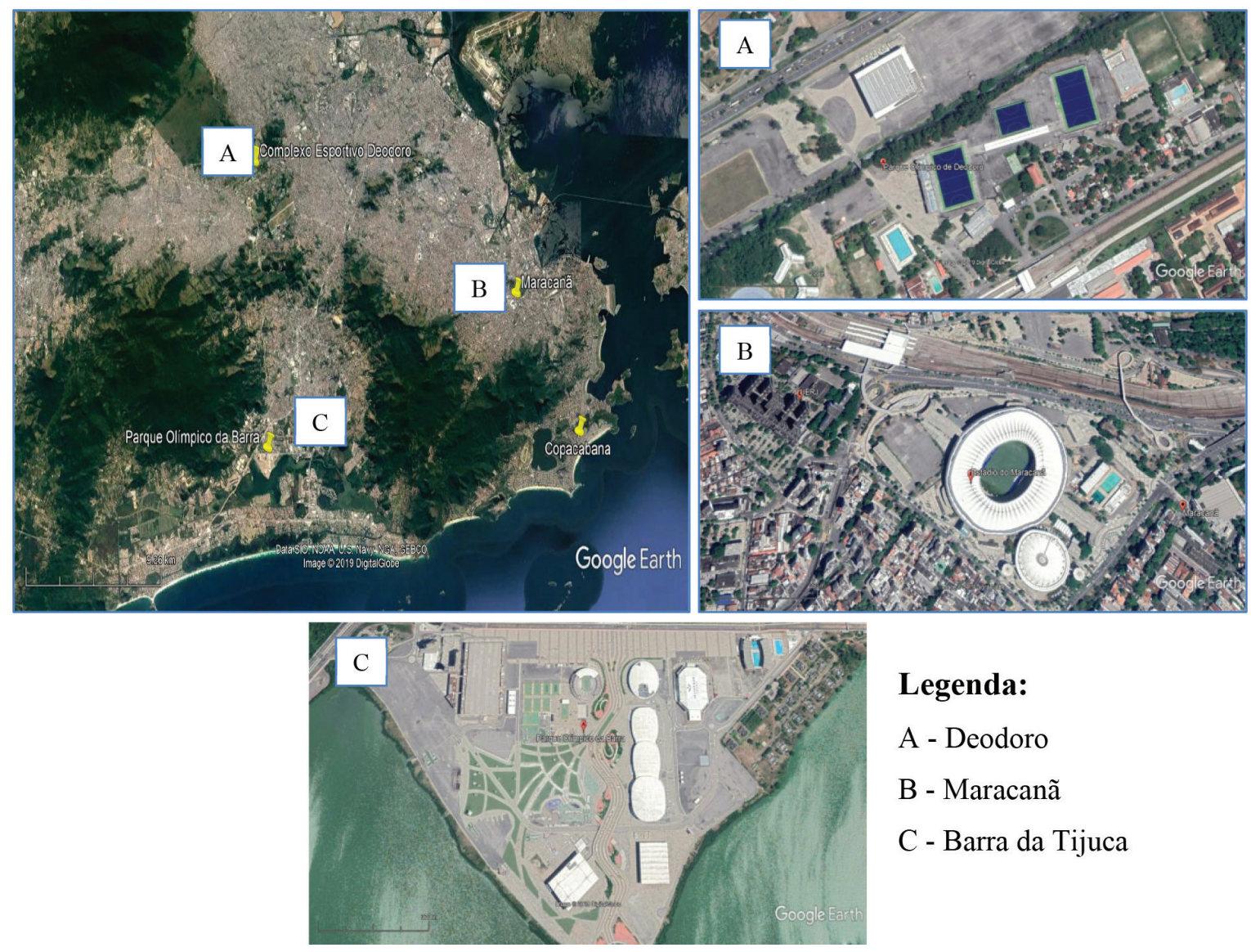

Legenda:
A - Deodoro
B - Maracanã
C - Barra da Tijuca

Figura 1. Mapa das áreas de amostragem em três regiões do Rio de Janeiro 
No processo de extração, o cartucho é quebrado em duas partes, separando o carvão do leito principal e do leito de segurança e estes são transferidos para vials de 2,0 mL, submersos em um recipiente contendo água gelada para evitar a perda por evaporação de compostos leves. ${ }^{4,15} \mathrm{Em}$ cada vial foi adicionado $1,00 \mathrm{~mL}$ de diclorometano grau HPLC a $-20^{\circ} \mathrm{C}$. Os vials lacrados foram colocados em banho de ultrassom por 10 minutos. Após esse período, os vials foram mantidos em repouso para que houvesse a decantação do carvão, deixando o sobrenadante límpido para posterior análise cromatográfica.

As análises químicas foram realizadas por cromatografia gasosa acoplado à espectrometria de massas (CG-EM), utilizando um cromatógrafo Varian modelo 450GC acoplado a um espectrômetro de massas Varian MS220. As condições cromatográficas foram otimizadas para a injeção de $1,0 \mu \mathrm{L}$, sem divisão de fluxo, com temperatura do injetor a $200{ }^{\circ} \mathrm{C}$, a fase móvel foi o Hélio 5.0 a 2,0 mL min ${ }^{-1}$. A coluna utilizada foi a Restek Rxi (30 m x 0,25 mm x $0,25 \mu \mathrm{m})$ e a programação de temperatura do forno iniciou com $40^{\circ} \mathrm{C}$ por $2 \mathrm{~min}$, com rampa de aquecimento de $10^{\circ} \mathrm{C} \mathrm{min}{ }^{-1}$ até a temperatura final de $200{ }^{\circ} \mathrm{C}$, mantida por 2 minutos. ${ }^{6,14,15,25}$

Os íons m/z 77-78, 91-92 e 103-104 foram selecionados para a identificação dos BTEX por comparação dos tempos de retenção dos analitos, das amostras e dos padrões. A curva analítica para cada composto foi preparada à partir da diluição de um padrão analítico (Supelco BTEX Mix 47993) com os 6 BTEX. O coeficiente de determinação $\left(\mathrm{R}^{2}\right)$ para cada composto foi superior a 0,98 . As concentrações dos padrões variaram de 0,1 a $20,0 \mathrm{mg} \mathrm{L}^{-1}$ e os padrões foram injetados em 5 níveis de concentração, com injeções em duplicata. Os limites de quantificação (LQ) medidos foram de $18 \mu \mathrm{g} \mathrm{L}^{-1}$ para o benzeno e $12 \mu \mathrm{g} \mathrm{L}^{-1}$ para os demais compostos e os limites de detecção (LD) correspondem a 1/3 dos valores de LQ.

\section{Riscos para a saúde humana}

As concentrações de BTEX foram usadas para avaliar a exposição não ocupacional a esses poluentes através da inalação de ar num ambiente externo. ${ }^{26}$ Nesse sentido, foi utilizado o Guia de Avaliação de Risco (Risk Assessment Guidance for Superfund) desenvolvido pela U.S. EPA. ${ }^{27}$ A concentração de exposição (EC) foi calculada conforme a Equação 1:

$$
\mathrm{EC}=\frac{\mathrm{CA} \times \mathrm{ET} \times \mathrm{FE} \times \mathrm{ED}}{\mathrm{AT}}
$$

em que EC é a concentração de exposição $\left(\mu \mathrm{g} \mathrm{m}^{-3}\right)$, CA é a concentração de cada BTEX $\left(\mu \mathrm{g} \mathrm{m}^{-3}\right)$, ET é tempo de exposição (8 horas dia $\left.^{-1}\right)$, FE é a frequência de exposição ( 250 dias $\left.^{2} o^{-1}\right)$, ED é a duração da exposição (30 anos) e AT é o tempo médio (70 anos de tempo de vida $\times 365$ dias ano $\left.{ }^{-1} \times 24 \mathrm{~h} \mathrm{dia}^{-1}\right)$

A Equação 2 representa o coeficiente de risco não carcinogênico (HQ), onde EC é calculado para cada BTEX $\left(\mu \mathrm{g} \mathrm{m}^{-3}\right)$ e o valor de toxicidade (ou RFC), expressa em $\mathrm{mg} \mathrm{m}^{-3}$, é a concentração de referência de inalação. ${ }^{27}$

$$
\mathrm{HQ}=\frac{\mathrm{EC}}{\text { Valor de toxicidade } \times 1000 \mu \mathrm{g} \mathrm{mg}^{-1}}
$$

A Equação 3 mostra o cálculo para o risco carcinogênico (CR), através da multiplicação entre o EC calculado para cada composto $\left(\mu \mathrm{g} \mathrm{m}^{-3}\right)$ pela unidade de inalação de referência (IUR), expressa em volume de ar por unidade de tempo. ${ }^{27}$

$$
\mathrm{CR}=\mathrm{EC} \times \mathrm{IUR}
$$

O risco carcinogênico pode ser considerado indiferente quando for menor que $1,00 \times 10^{-6}$, mas é considerado significativo quando é maior que $1,00 \times 10^{-3}$; já o risco não carcinogênico pode ser considerado indiferente quando for igual a $1,0.27$

\section{Escalas de reatividade}

A reatividade cinética foi obtida através do produto entre a concentração média de cada composto, em cada área, e o seu respectivo valor de $\mathrm{k}_{\mathrm{OH}}$. Após o cálculo deste produto, foi realizada a análise da contribuição, em percentagem, de cada BTEX.7,11

O cálculo da reatividade mecanística foi obtido pelo produto entre a concentração de cada composto, em cada área, e o seu respectivo coeficiente adimensional MIR, MOIR e EBIR, permitindo o estabelecimento de três diferentes escalas de reatividade, para os três diferentes locais, bem como a contribuição de cada composto em percentagem. ${ }^{7,11}$

\section{RESULTADOS E DISCUSSÃO}

Os resultados da estatística descritiva para cada local de coleta são mostrados nos boxplots da Figura 2. O intervalo de confiança de $95 \%$ foi utilizado de acordo com o número de amostras de cada composto coletado em cada área.

Comparando as concentrações médias nas três áreas de estudo, os maiores valores foram observados na região do Maracanã, onde o benzeno medido foi 1,37 e 1,89 vezes acima das concentrações medidas em Deodoro e na Barra respectivamente. O tolueno está 2,42 vezes acima do valor observado em Deodoro e 3,16 vezes acima do medido na Barra, assim como o etilbenzeno que apresentou razões superiores em 8,39 e 12,74 vezes, respectivamente, para Deodoro e Barra. Os isômeros $m+p$-xilenos tiveram concentrações 5,85 e 5,43 vezes maiores na área Maracanã comparadas com as áreas Deodoro e Barra. O o-xileno no Maracanã apresentou as maiores diferenças nas razões entre as áreas, sendo 22,3 vezes maior que em Deodoro e 20,27 vezes maior do que na Barra.

Uma possível explicação para as mais altas concentrações na área do Maracanã comparadas às outras áreas desse trabalho seria a proximidade de vias de intenso tráfego de veículos e a urbanização acentuada da região com muitos prédios, o que dificulta a circulação dos ventos e a dispersão dos poluentes nesta região. ${ }^{28}$

A Figura 3 apresenta a distribuição percentual dos BTEX, indicando o tolueno como o composto mais abundante nos três locais, com 54,3\% na Barra da Tijuca, 57,4\% em Deodoro e 38,1\% na área do Maracanã do total de BTEX (média de 41,3\% para o total de amostras). As áreas de Deodoro e da Barra apresentaram uma leve similaridade nos perfis de distribuição, todavia, a área Maracanã possui um perfil diferente das demais. As emissões nos três locais são predominantemente veiculares. No bairro do Maracanã foram observadas as menores velocidades médias e os maiores congestionamentos nos dias das coletas das amostras. As diferenças topográficas e de condições de dispersão dos poluentes entre os locais também irão contribuir para a diferença nos perfis de concentração medidos. ${ }^{20,28}$

As razões entre os BTEX podem fornecer informações sobre as suas fontes de emissão de acordo com Martins et al.,${ }^{29}$ sendo que a razão benzeno/tolueno $(\mathrm{B} / \mathrm{T})$ pode ser usada como um indicativo de emissão predominantemente veicular para intervalos de razão entre 0,2 e 0,5 . Os mesmos autores avaliaram as razões B/T no centro da cidade de São Paulo que variaram de 0,18 a 0,49 , com média de $0,32 \pm 0,10$, indicando que a fonte de emissão destes compostos é predominantemente veicular. Já para este trabalho, as razões médias obtidas foram de 0,12 $\pm 0,08$ (Maracanã), 0,37 $\pm 0,50$ (Barra da Tijuca) 

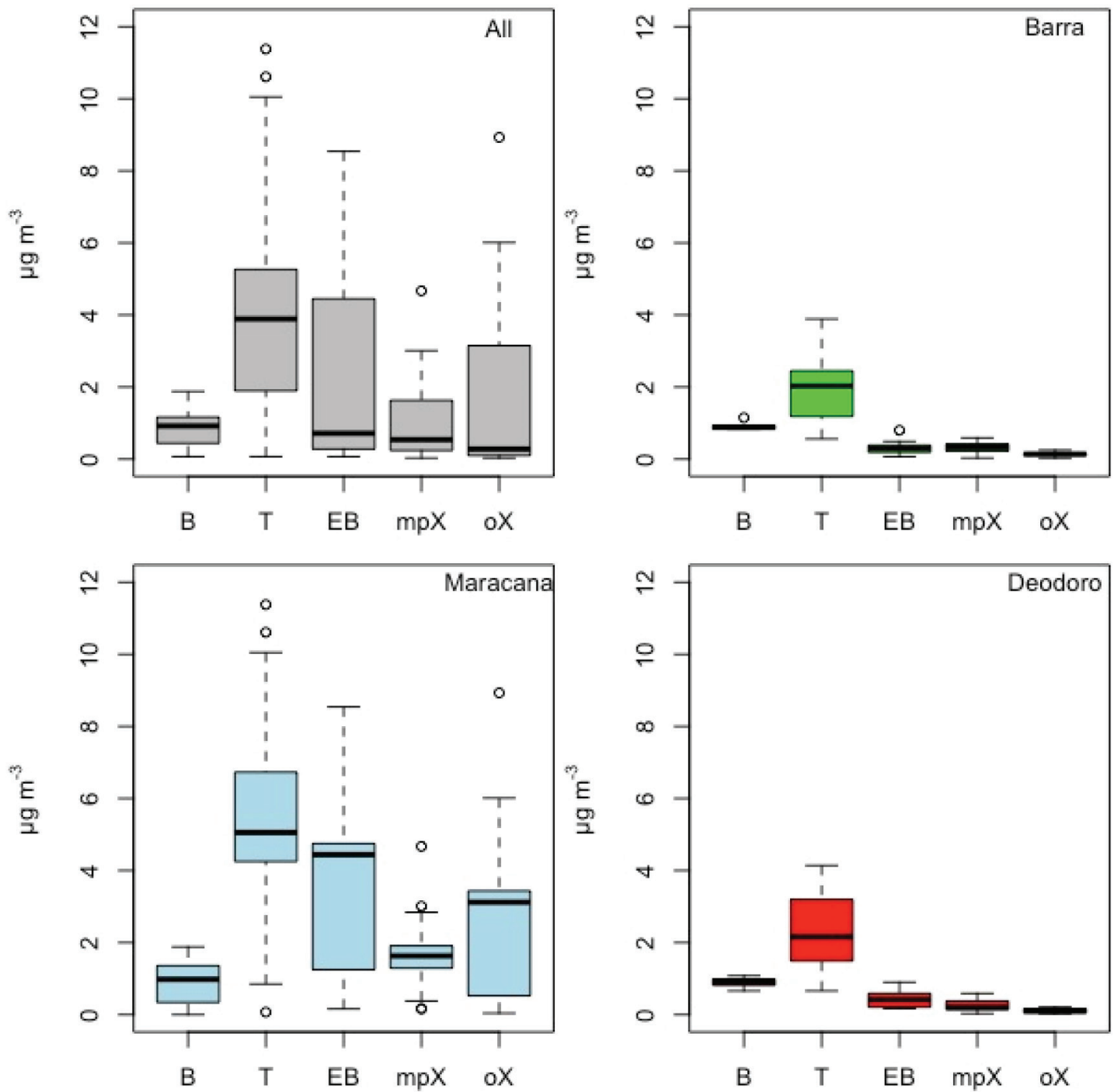

Figura 2. Boxplot das concentrações (em $\mu \mathrm{g} \mathrm{m}^{-3}$ ) das amostras agrupadas na Barra da Tijuca, Maracanã e Deodoro

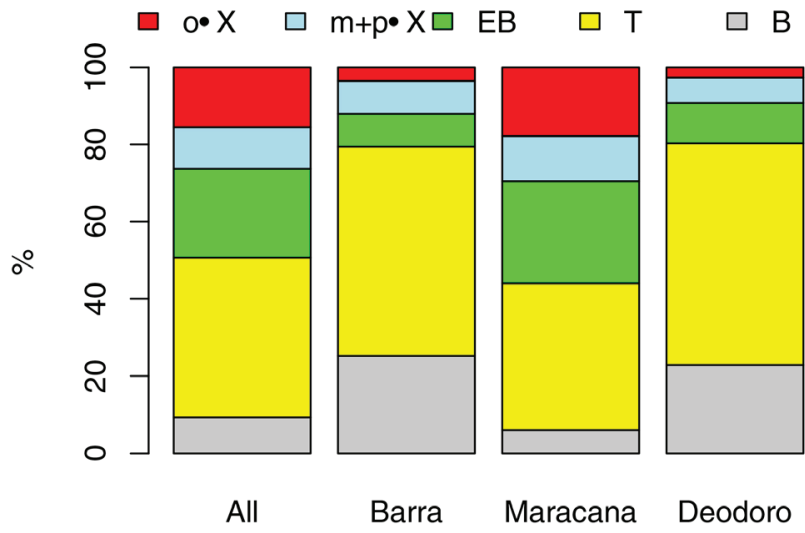

Figura 3. Distribuição percentual dos BTEX para as três áreas de estudo e dados agrupados

e 0,23 $\pm 0,23$ (Deodoro), sugerindo o mesmo tipo de fonte de emissão encontrada por Martins et al. ${ }^{29}$

Segundo Fernandes et al. ${ }^{30}$ a razão B/T de 0,1 pode indicar um alto valor de aromáticos contido nos combustíveis e, consequentemente, o aumento da emissão de tolueno pelos automóveis. Os autores encontraram em seu estudo, a razão $\mathrm{B} / \mathrm{T}$ variando entre 0,2 e 0,3 no Alto da Boa Vista, Ilha do Governador e centro comercial da cidade do Rio de Janeiro, com exceção da Avenida Brasil que apresentou resultado próximo a 0,5 .

As concentrações observadas em Pequim por Liu et al. ${ }^{31}$ foram coletadas semanas antes do início dos jogos olímpicos da China e registraram valores mais altos de benzeno quando comparados a este trabalho. Mesmo durante o evento e com medidas governamentais para a redução do tráfego e de produção nas indústrias, as médias registradas em Pequim foram de $2,37 \mu \mathrm{g} \mathrm{m}^{-3}$ para o benzeno e $3,97 \mu \mathrm{g} \mathrm{m}^{-3}$ para o tolueno.

A Tabela 1 apresenta os valores dos COV avaliados neste trabalho e os compara com estudos realizados em outros locais do Rio de Janeiro. Como as concentrações da área do Maracanã foram as mais expressivas neste trabalho para a maioria dos COV, elas foram escolhidas para representar este estudo.

As concentrações de BTEX analisadas por Geraldino et al. ${ }^{32}$ no bairro de Bangu, na zona oeste do Rio de Janeiro, apresentaram resultados relativamente maiores para o benzeno ( 2,18 vezes), tolueno ( 1,23 vezes) e o-xileno ( 1,17 vezes) quando comparados com os resultados obtidos neste trabalho para o bairro do Maracanã. $\mathrm{O}$ autor concluiu que montanhas na parte sul e norte da região oeste podem contribuir para o aumento da temperatura da superfície da terra e o acúmulo de poluentes na região, justificando as altas concentrações observadas.

Martins et al. ${ }^{33}$ avaliaram as concentrações de aldeídos e BTEX durante 12 meses na Tijuca, bairro da zona norte do Rio de Janeiro. 
Os dados são consideravelmente mais altos, destacando o $\mathrm{m}+\mathrm{p}$ xileno, sendo este seis vezes maior que o valor encontrado no bairro do Maracanã. Esses dados podem ser atribuídos às condições topográficas e meteorológicas da região, além do grande fluxo veicular.

Tabela 1. Comparação das concentrações médias $\left(\mathrm{em} \mu \mathrm{g} \mathrm{m}^{-3}\right.$ ) dos COV analisados nas áreas deste estudo com outros trabalhos realizados na cidade do Rio de Janeiro

\begin{tabular}{lccccc}
\hline & $\mathrm{B}$ & $\mathrm{T}$ & $\mathrm{EB}$ & $\mathrm{m}+\mathrm{p}-\mathrm{X}$ & $\mathrm{o}-\mathrm{X}$ \\
\hline Deodoro $^{(\mathrm{a})}$ & 0,51 & 2,24 & 0,41 & 0,26 & 0,10 \\
Barra $^{(\mathrm{a})}$ & 0,37 & 1,72 & 0,27 & 0,28 & 0,11 \\
Maracanã $^{(\mathrm{a})}$ & 0,70 & 5,43 & 3,44 & 1,52 & 2,23 \\
Bangu $^{(\mathrm{b})}$ & 1,53 & 6,70 & 1,54 & 1,56 & 2,61 \\
Tijuca $^{(\mathrm{c})}$ & 1,10 & 4,8 & 3,6 & 10,40 & 3,0 \\
Rio de Janeiro $^{(\mathrm{d})}$ & 29,7 & 47,7 & 23,3 & 46,9 & 14,3 \\
\hline
\end{tabular}

(a) Este estudo; (b) Geraldino et al.;32 (c) Martins et al.;3 (d) Corrêa et $_{\text {al. }}{ }^{34}$

Já os valores médios apresentados para as 48 amostras de BTEX no entorno de postos de gasolina da cidade do Rio de Janeiro para os anos de 2008 e 2009 analisados por Corrêa et al $^{34}$ apresentam valores mais elevados que os reportados neste estudo para ar ambiente, corroborando com a influência das emissões evaporativas majoritárias.

All

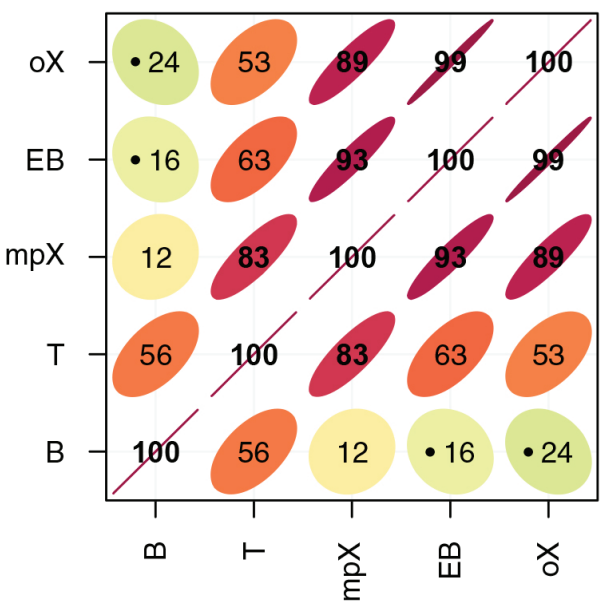

Maracana

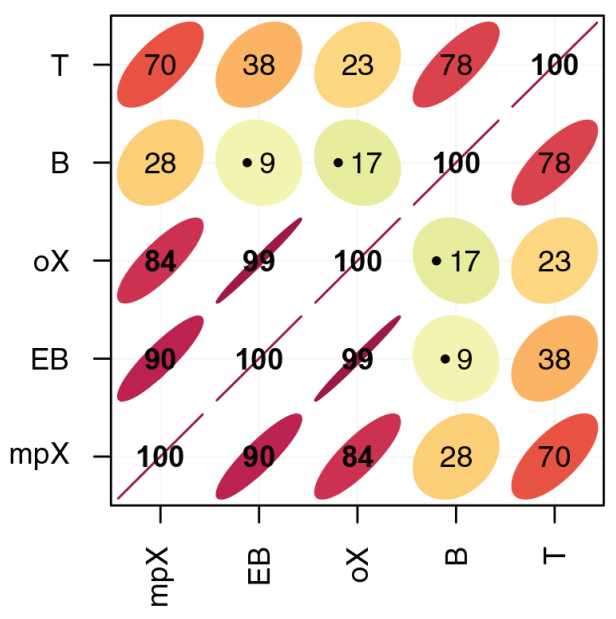

Foram calculadas as correlações de Pearson à partir das concentrações de BTEX, num intervalo de confiança de $95 \%$, para as três áreas de estudo durante todo o período de amostragem. A Figura 4 apresenta os resultados obtidos.

Analisando as correlações entre os BTEX pode-se observar que para Deodoro e Barra, todos os valores são positivos, com valores mais elevados para Deodoro. Isso pode sugerir que existam fontes de BTEX similares ou estes compostos na região sofrem os mesmos processos de formação e/ou remoção. Já para a região do Maracanã existem correlações negativas entre benzeno e o-xileno.

Escalas de reatividade

No Material Suplementar são mostrados os resultados das reatividades cinética e mecanística (Tabela 1S) dos BTEX em cada local analisado.

Como apresentado nas Figuras 5 e 6 , o tolueno e $m+p$-xileno foram os compostos de maior potencial de formação de ozônio na escala de reatividade do $\mathrm{OH}$ em todos os locais analisados. $\mathrm{O}$ tolueno foi o composto mais abundante nos locais de amostragem, o que justifica a mais elevada reatividade com os radicais $\mathrm{OH}$.

Os resultados apontaram semelhanças nas áreas Barra e Deodoro em relação à ordem das escalas de reatividade MOIR e EBIR. As contribuições mais altas de $\mathrm{m}+\mathrm{p}$-xilenos nas três escalas também foram observadas na Barra e em Deodoro. Já a área Maracanã possui
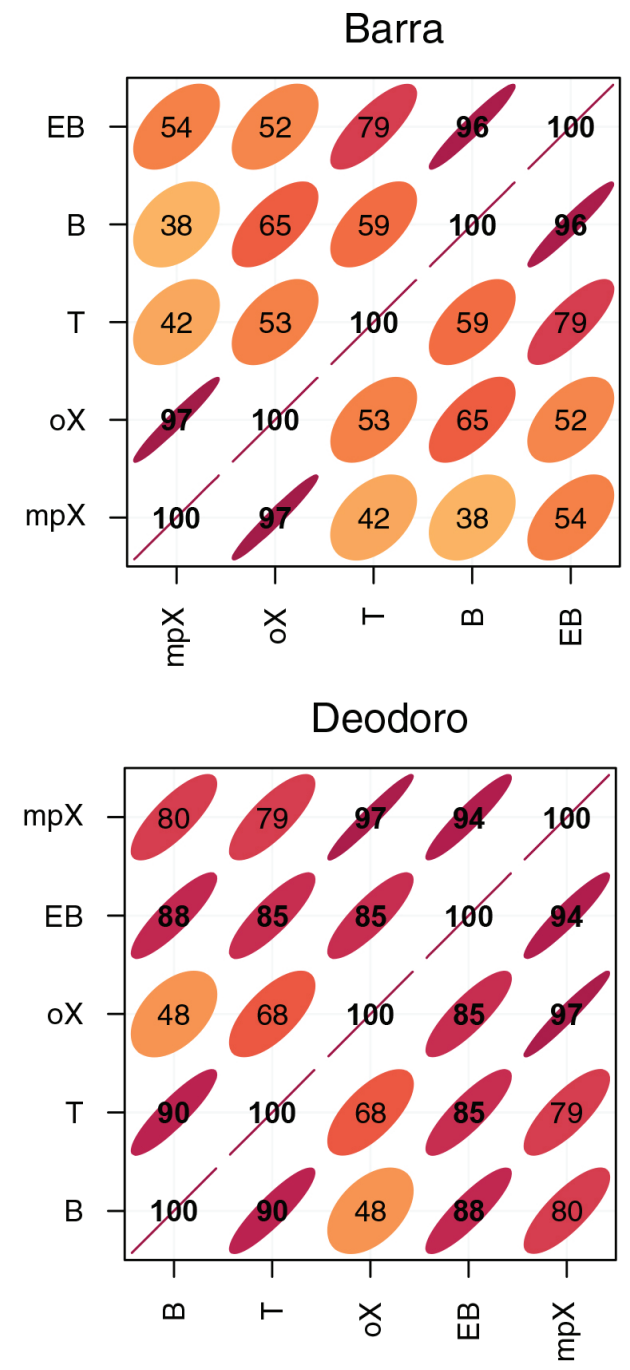

Figura 4. Matriz de correlação das concentrações de BTEX das três áreas de estudo e com todos os dados 


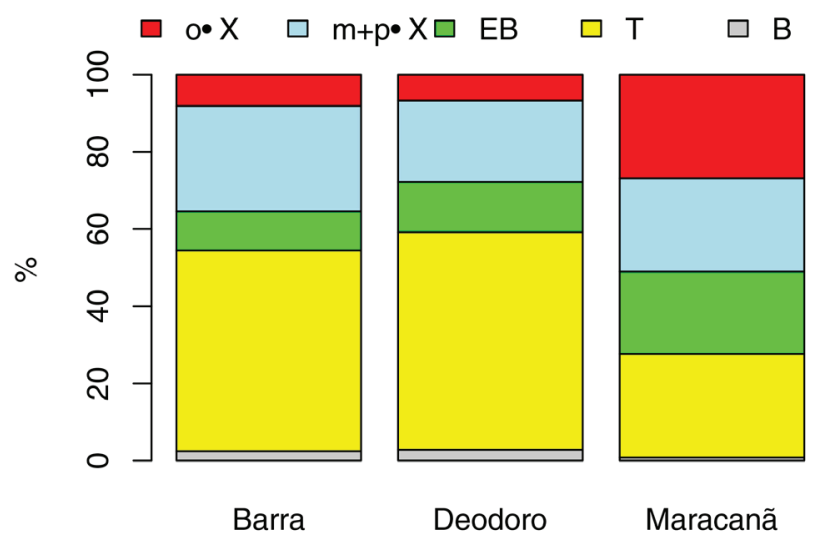

Figura 5. Contribuição de BTEX quanto à reatividade cinética para as três áreas de estudo

características diferentes das demais, onde a maioria dos BTEX, com exceção do benzeno, contribuem para a formação do ozônio nas três escalas.

Os BTEX possuem diferentes tempos de residência na atmosfera, onde o processo de degradação desses compostos na atmosfera ocorre através de reações com os radicais $\mathrm{OH}$. O benzeno possui maior tempo de vida na atmosfera, sendo portanto o menos reativo, enquanto que o tolueno permanece menos tempo na atmosfera, logo possui maior reatividade quando comparado com o benzeno. Já os xilenos são os compostos mais reativos, sendo degradados rapidamente em questão de horas. ${ }^{35}$

Geraldino et al.${ }^{32}$ se basearam nas concentrações encontradas em seu trabalho para a obtenção da escala MIR para o bairro de Bangu, Rio de Janeiro. Os COV analisados que influenciaram na formação do ozônio seguiram a seguinte ordem de reatividade: tolueno, $\mathrm{m}+\mathrm{p}$ -xilenos, o-xileno, etilbenzeno e benzeno.

Castro et al. ${ }^{4}$ avaliaram os BTEX em local confinado (estacionamento de um shopping center no Rio de Janeiro) e obtiveram resultados de reatividade na escala MIR de benzeno ( $2 \%)$, tolueno $(38,2 \%)$, etilbenzeno $(5,9 \%), \mathrm{m}+\mathrm{p}$-xilenos $(47,3 \%)$ e o-xileno $(10,2 \%)$, onde puderam concluir que os compostos que mais contribuíram para a formação de ozônio foram os $\mathrm{m}+\mathrm{p}$-xilenos e o tolueno.

\section{Risco à saúde humana}

A partir das concentrações de BTEX, foram obtidos os resultados dos riscos carcinogênicos e não carcinogênicos referente à exposição destes compostos para as três áreas de estudo. Os valores médios, máximos e mínimos para esses riscos observados nas três áreas são apresentados na Tabela 2.

O risco carcinogênico foi avaliado a partir dos resultados de benzeno. O risco médio de câncer nas áreas Barra, Deodoro e Maracanã, para uma estimativa de 70 anos de vida, foram estimados em $2,82 \times 10^{-7}, 3,89 \times 10^{-7}$ e $5,34 \times 10^{-7}$, respectivamente, sendo esses valores inferiores aos encontrados por Vilavert et al. ${ }^{26}$ que analisaram pontos próximos a um aterro sanitário na Espanha e encontraram valores na ordem de grandeza de $10^{-6}$. Entretanto, os valores observados neste trabalho foram superiores ao risco carcinogênico avaliado em um aterro na Itália, segundo Palmiotto et al,.$^{36}$ onde os autores obtiveram resultados na ordem de grandeza entre $10^{-8}$ e $10^{-9}$.

Os riscos carcinogênicos observados nas três áreas deste trabalho podem ser considerados indiferentes, pois são menores que $1,00 \times 10^{-6}$. Valores acima de $1,00 \times 10^{-3}$ são considerados significativos, indicando algum efeito adverso à saúde, segundo o Guia de Avaliação de Risco U.S. EPA. ${ }^{27}$

O Quociente de Risco (HQ) é um parâmetro que demonstra os efeitos não carcinogênicos à saúde humana devido à exposição a um composto. Quando HQ é menor que 1, pode indicar que nenhum efeito adverso à saúde humana será observado durante o tempo de exposição. Contudo, valores acima de 1 possibilitam prováveis efeitos adversos à saúde humana durante o tempo de exposição ao poluente. Portanto, ao comparar valores de risco não carcinogênicos nas áreas de estudo, foi observado que todos os valores de BTEX estavam menores que 1 , indicando que não há risco.

\section{CONCLUSÕES}

As concentrações de BTEX na área da Barra da Tijuca foram, em sua maioria, menores que as áreas do Maracanã e Deodoro. Apesar do tráfego intenso, a proximidade com o mar mantém a circulação dos ventos e, consequentemente, contribui com a dispersão dos poluentes atmosféricos.

O tolueno é o composto mais abundante entre os BTEX. A razão $\mathrm{B} / \mathrm{T}$ indica que a fonte de emissão destes compostos nas três áreas estudadas é predominantemente veicular. Esse resultado confirma a situação geral em que se encontra a Região Metropolitana do Rio de Janeiro (RMRJ), bem como em outras grandes cidades ao redor do mundo, onde o aumento da frota veicular e a consequente queima de combustíveis fósseis é a maior fonte de emissão de poluentes.

Os resultados das escalas de reatividade $\mathrm{k}_{\mathrm{OH}}$, MIR, MOIR e EBIR para os BTEX avaliados neste trabalho foram relativamente diferentes

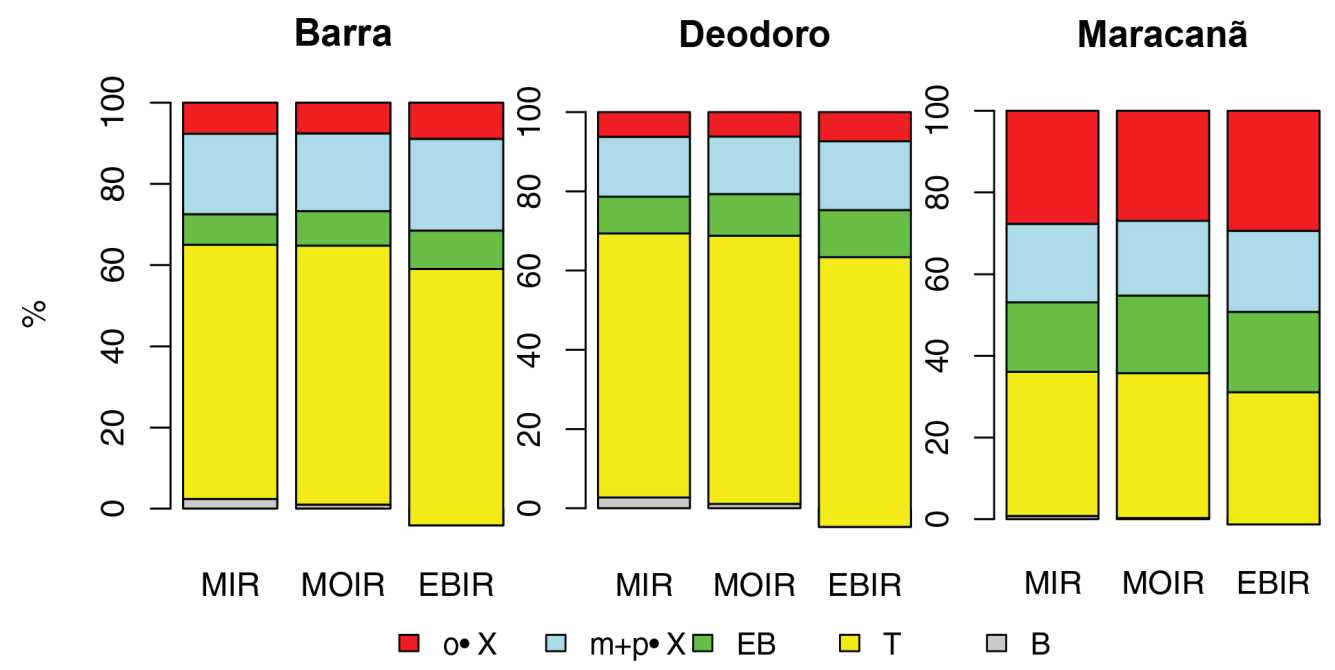

Figura 6. Contribuição de BTEX quanto à reatividade mecanística para as três áreas de estudo 
Tabela 2. Risco carcinogênico (RC) e não-carcinogênico (RNC) para exposição de BTEX

\begin{tabular}{|c|c|c|c|c|c|c|}
\hline & \multicolumn{3}{|c|}{ RNC } & \multicolumn{3}{|c|}{$\mathrm{RC}$} \\
\hline & Média & Máximo & Mínimo & Média & Máximo & Mínimo \\
\hline \multicolumn{7}{|c|}{ Barra } \\
\hline B & $1,21 \times 10^{-3}$ & $3,75 \times 10^{-3}$ & $<\mathrm{LQ}^{*}$ & $2,82 \times 10^{-7}$ & $8,78 \times 10^{-7}$ & $<\mathrm{LQ}$ \\
\hline $\mathrm{T}$ & $3,37 \times 10^{-5}$ & $7,61 \times 10^{-5}$ & $<\mathrm{LQ}$ & - & - & - \\
\hline $\mathrm{EB}$ & $2,64 \times 10^{-5}$ & $7,83 \times 10^{-5}$ & $<\mathrm{LQ}$ & - & - & - \\
\hline$m+p-X$ & $2,74 \times 10^{-4}$ & $5,77 \times 10^{-4}$ & $<\mathrm{LQ}$ & - & - & - \\
\hline$o-X$ & $1,08 \times 10^{-4}$ & $2,45 \times 10^{-4}$ & $<\mathrm{LQ}$ & - & - & - \\
\hline \multicolumn{7}{|c|}{ Deodoro } \\
\hline B & $1,66 \times 10^{-3}$ & $3,52 \times 10^{-3}$ & $<\mathrm{LQ}$ & $3,89 \times 10^{-7}$ & $8,24 \times 10^{-7}$ & $<\mathrm{LQ}$ \\
\hline $\mathrm{T}$ & $4,38 \times 10^{-5}$ & $8,10 \times 10^{-5}$ & $1,29 \times 10^{-5}$ & - & - & - \\
\hline E & $4,01 \times 10^{-5}$ & $8,81 \times 10^{-5}$ & $1,66 \times 10^{-5}$ & - & - & - \\
\hline$m+p-X$ & $2,54 \times 10^{-4}$ & $5,77 \times 10^{-4}$ & $<\mathrm{LQ}$ & - & - & - \\
\hline$o-X$ & $1,08 \times 10^{-4}$ & $2,05 \times 10^{-4}$ & $<\mathrm{LQ}$ & - & - & - \\
\hline \multicolumn{7}{|c|}{ Maracanã } \\
\hline B & $2,28 \times 10^{-3}$ & $5,68 \times 10^{-3}$ & $<\mathrm{LQ}$ & $5,34 \times 10^{-7}$ & $1,33 \times 10^{-6}$ & $<\mathrm{LQ}$ \\
\hline $\mathrm{T}$ & $1,06 \times 10^{-4}$ & $2,08 \times 10^{-4}$ & $1,66 \times 10^{-5}$ & - & - & - \\
\hline EB & $3,37 \times 10^{-4}$ & $8,36 \times 10^{-4}$ & $1,57 \times 10^{-5}$ & - & - & - \\
\hline$m+p-X$ & $1,49 \times 10^{-3}$ & $2,78 \times 10^{-3}$ & $1,57 \times 10^{-4}$ & - & - & - \\
\hline$o-X$ & $2,18 \times 10^{-3}$ & $5,88 \times 10^{-3}$ & $3,91 \times 10^{-5}$ & - & - & - \\
\hline
\end{tabular}

$*<\mathrm{LQ}=$ abaixo do limite de quantificação.

para as três áreas estudadas. Como os cenários usados para representar as escalas foram baseados em cidades dos Estados Unidos, sugerese que mais estudos específicos desta escala em relação aos COV encontrados aqui no Brasil sejam feitos, uma vez que cada região/ país têm as suas particularidades, como a geografia, clima, tráfego de veículos e tipo de combustíveis utilizados. Esses fatores externos influenciam e devem ser considerados na determinação das escalas, pois o mesmo COV pode diferir a sua concentração em regiões diferentes e resultar em distintas razões COV/NOx.

Os riscos carcinogênicos e não carcinogênicos pela exposição de BTEX nas áreas analisadas, dentro das probabilidades aplicadas para uma exposição de ar exterior, não causam efeitos diretos à saúde da população ao longo do tempo de vida estimada, possivelmente pela facilidade de dispersão dos poluentes atmosféricos através da circulação dos ventos.

A sugestão para trabalhos futuros é que os COV continuem sendo monitorados no Rio de Janeiro, a fim de reforçar a necessidade de inclusão de compostos bastante relevantes em termos de toxicidade na legislação brasileira, uma vez que a Resolução CONAMA 03/1990 foi recentemente atualizada (CONAMA 491) no ano de 2018 e não incluiu esses compostos.

\section{MATERIAL SUPLEMENTAR}

A Tabela 1S (reatividades cinética e mecanística) e as Figuras 1S-2S (concentrações médias horárias de ozônio) utilizadas neste trabalho estão disponíveis em http://quimicanova.sbq.org.br, na forma de arquivo PDF, com acesso livre.

\section{AGRADECIMENTOS}

Os autores agradecem o apoio financeiro da FAPERJ e do CNPq. Graphical Abstract: elaborado por Freepik.

\section{REFERÊNCIAS}

1. Seinfeld, J. H.; Pandis, S. N.; Atmospheric Chemistry and Physics: from air pollution to climate change, $2^{\text {nd }}$ ed., John Wiley \& Sons: New York, 2006.

2. SMAC; Relatório da Rede MonitorAr Rio 2011 - 2012. Qualidade do Ar na Cidade do Rio de Janeiro, Prefeitura do Rio de Janeiro, 2012.

3. Masih, J.; Masih, A.; Kulshrestha, A.; Singhvi, R.; Taneja, A. J. Hazard. Mater. 2010, 177, 190.

4. Castro, B. P.; Machado, G. S.; Bauerfeldt, G. F.; Fortes, J. D. N.; Martins, E. M.; Atmos. Environ. 2015, 104, 22.

5. Martins, E. M.; Nunes, A. C. L.; Corrêa, S. M.; J. Braz. Chem. Soc. 2015b, 26, 1967.

6. Martins, E. M.; Silva, D. B. N.; Corrêa, S. M.; Environ. Monit. Assess. 2016a, 188, 289.

7. Carter, W. P. L.; J. Air Waste Manage. Assoc. 1994, 44, 881.

8. Wang, L.; Milford, J. B.; Carter, W. P. L.; Atmos. Environ. 2000, 34, 4349.

9. Silva, C. M.; Tese de Doutorado, Universidade do Estado do Rio de Janeiro, Brasil, 2016

10. Geraldino, C. G. P.; Dissertação de Mestrado, Universidade do Estado do Rio de Janeiro, Brasil, 2017.

11. http://www.engr.ucr.edu/ carter/SAPRC/, acessada em maio de 2020.

12. http://cfpub.epa.gov/ncea/iris/iris_documents/documents/ toxreviews/0276tr.pdf, acessada em maio de 2020.

13. Lim, S. K.; Shin, H. S.; Yoon, K. S.; Kwack, S. J.; Yoon Mi Um, Y. M.; Hyeon, J. H.; Kwak, H. M.; Kim, J. Y.; Kim, T. H.; Kim, Y. J.; Roh, T. H.; Lim, D. S.; Shin, M. K.; Choi, S. M.; Kim, H. S.; Lee, B. M.; J. Toxicol. Environ. Health, Part A 2014, 77, 1502.

14. Martins, E. M.; Borba, P. F. de S.; dos Santos, N. E.; dos Reis, P. T. B.; Silveira, R. S.; Corrêa, S. M. Environ. Monit. Assess. 2016b, 188, 608.

15. Martins, E. M.; Borba, P. F. de S.; dos Santos, N. E.; dos Reis, P. T. B.; Silveira, R. S.; Israel, F.; Ferraz, E. R. A.; Fernandes, A. da S.; Muniz, 
R. da S.; Matos, I. B. de S.; Corrêa, S. M.; International Journal of Environmental Impacts 2019, 2, 174.

16. Vallero, D. A.; Fundamentals of air pollution, $5^{\text {th }}$ ed., Elsevier: Oxford, 2014.

17. IARC Monographs, Programme on the Evaluation of the Carcinogenic Risk of Chemicals to Human, 2018, v.120.

18. Martins, E. M.; Monteiro, M.; Fortes, J. D. N.; Corrêa, S. M.; Quitério, S. L.; Castro, B. P.; Cadernos de Saúde Coletiva 2014, 22, 218.

19. https://cidades.ibge.gov.br/brasil/rj/rio-de-janeiro/panorama, acessada em maio de 2020.

20. Inea, Relatório da Qualidade do Ar, ano base 2015, Estado do Rio de Janeiro, 2016

21. https://infraestrutura.gov.br/component/content/article/115-portaldenatran/8559-frota-de-veiculos-2019.html, acessada em maio de 2020.

22. Hydrocarbons Aromatic: Method 1501. Manual of Analytical Methods, National Institute of Occupation Safety and Health, Cincinnati, 1997

23. Caselli, M.; Gennaro, G.; Marzocca, A.; Trizio, L.; Tutino, M.; Chemosphere 2010, 81, 306.

24. Buczynska, A. J.; Krata, A.; Stranger, M.; Godoi, A. F. L.; KontozovaDeutsch, V.; Bencs, L.; Naveau, I.; Roekens, E.; Grieken, R. V.; Atmos. Environ. 2009, 43, 311.

25. De Sá Borba, P. F.; Martins, E. M.; Ritter, E.; Corrêa, S. M.; Bull. Environ. Contam. Toxicol. 2017, 98, 624.

26. Vilavert, L.; Nadal, M.; Figueras, M. J.; Domingo, J. L.; Environ. Sci. Pollut. Res. 2012, 19, 96.
27. Risk assessment guidance for superfund volume I: human health evaluation manual (part $F$, supplemental guidance for inhalation risk assessment), United States Environmental Protection Agency. Office of Superfund Remediation and Technology Innovation, EPA540-R-070-002, 2009

28. http://www.inea.rj.gov.br/cs/groups/public/@inter_vpres_geiat/ documents/document/zwew/mti3/ edisp/inea0127611.pdf, acessada em maio de 2020

29. Martins, L. D.; Andrade, M. F.; Ynoue, R. Y.; Albuquerque, E. L.; Tomaz, E.; Vasconcellos, P. C.; Quim. Nova 2008, 31, 2009.

30. Fernandes, M. B.; Brickus, L. S. R.; Moreira, J. C.; Cardoso, J. N.; Chemosphere 2002, 47, 417.

31. Liu, J.; Mu, Y.; Zhang, Y.; Zhang, Z.; Wang, X.; Liu, Y.; Sun, Z.; Sci. Total Environ. 2009, 408, 109.

32. Geraldino, C. G. P.; Martins, E. M.; da Silva, C. M.; Arbilla, G.; Bull. Environ. Contam. Toxicol. 2017, 98, 632.

33. Martins, E. M.; Arbilla, G.; Bauerfeldt, G. F.; de Paula, M.; Chemosphere 2007, 67, 2096.

34. Corrêa, S. M.; Arbilla, G.; Marques, M. R. C.; Oliveira, K. M. P. G.; Atmos. Pollut. Res. 2012, 3, 163-169.

35. Cruz, L. P. S.; Santos, D. F.; dos Santos, I. F.; Gomes, I. V. S.; Santos, A. V. S.; Souza, K. S. P. P.; Microchem. J. 2020, 152, 104265.

36. Palmiotto, M.; Fattore, E.; Paiano, V.; Celeste, G.; Colombo, A.; Davoli, E.; Environ. Int. 2014, 8, 16. 